\title{
SOCIAL REGIONAL DEVELOPMENT - TRENDS AND PERSPECTIVES (THE BULGARIAN CASE)
}

\author{
DOI: http://dx.doi.org/10.18509/GBP.2020.70 \\ UDC: 331.5:303.6/.7(497.2)"2014/2019" \\ 37:303.6/.7(497.2)"2014/2019" \\ 614:303.6/.7(497.2)"2014/2019"
}

\author{
Kliment Naydenov \\ Sofia University "St. Kliment Ohridski, Bulgaria
}

\begin{abstract}
The state and quality of education in the country, national number one priority, are linked, on the one hand, to the demographic decline, and, on the other, to the small progress made in the reform of the system - the introduction of ICT, linking to the needs of business, accessibility, inclusion. Roma, lifelong learning and more. Statistics show that there is a well-developed health system in the country that is not functioning satisfactorily. Public health expenditure represents $4.4 \%$ of GDP and increases annually, with no significant effect and improvement in the system. The National Physical Education and Sport Policy considers sports activities in three directions - as part of the leisure time of the population; organized sport for all and sport for excellence. All of them have their important place in the development of the common physical culture; to maintain the good health status of the population on which the viability of the nation and the ability of the workforce depend; for developing sports talents and building national self-esteem. The labor market is crucial for the stability of the economy, for the social and social development of the country, for the standard and quality of life. The socio-economic basis for its development is a cumulative result of the good functioning of the systems in a number of areas demography, health care, education, pension system, business climate, incl. administrative service. A well-functioning labor market with a highly skilled workforce that can quickly acquire new skills is a prerequisite for a dynamic and competitive economy.
\end{abstract}

Keywords: social regional development, education, health care

\section{INTRODUCTION}

The state and quality of education in Bulgaria are linked, on the one hand, to the demographic decline and, on the other hand, to the small progress made in the reform of the system - the introduction of ICT, engagement with business needs, accessibility, Roma inclusion, learning throughout life and more. The number of students in the country decreases annually by nearly $1.5 \%$ and from 749094 in 2014 they drop to 730576 in 2017. Schools are closed, mainly in small settlements in peripheral areas. Compared to 2014, comprehensive schools have decreased by 108 due to closure or conversion. Although there is a symbolic decrease in the proportion of early school leavers, this proportion is still too high $-2.96 \%$. A major factor in the dropout rate is the presence of large Roma communities in some municipalities. The dropout students are mostly from smaller municipalities in the country where the poorer population is concentrated. Most students drop out of school because of the departure of their families abroad. Vocational education and training in the academic year 2017/2018 is implemented in 22 arts schools, 
24 sports schools, 359 vocational high schools and 29 vocational colleges with postsecondary education. The total number of schools is 434 , and the total number of students is 142.0 thousand. Compared to the 2014/2015 school year, vocational schools have decreased by 47 , with vocational schools being the most reduced. In the academic year 2017/2018, 1,048 students are enrolled in 38 private vocational high schools and private vocational colleges with post-secondary education, which is $0.7 \%$ of all students in vocational education[2].

\section{DATA \& METHODS}

In the article are used primary data and secondary sources and the use of both, which is termed triangulation, or dual methodology. Primary data, which we used, is the data collected by us, i.e. interview, observation, questionnaires. Secondary sources used in the article are data that already exists - previous research, official statistics, mass media products, government reports, web information, historical data and information.

\section{DISSCUSION}

The educational structure of the population is connected to the socio-economic structures, but also relevant to the mode of reproduction and its related indicator. It has been shown that increasing the level of education affect reproductive attitudes and behavior of people [6]. In economic sectors, the distribution of employment by educational level is more uneven, but in the service industries, the share of higher education employees is higher. The share of university graduates exceeds $60 \%$ in education, finance and insurance, healthcare and social activities, real estate operations, the creation and distribution of information and creative products and telecommunications, professional activities and research and government. Conversely, relatively more people with no secondary education are in water supply and sewerage, agriculture, forestry and fisheries, construction, extractive and processing industries and administrative and support activities. The problem of finding employment for low-educated people is also related to their low motivation and lack of career opportunities. The quality of education in the vocational school system does not yet meet the requirements of the labor market. Measures to stimulate training in the professions sought are imperative. A positive fact in vocational education is a certain increase in vocational school students. Job training (dual training) is a new opportunity to reintegrate early school leavers. Interest in this form of training is increasing. The most important long-term factors that have had an impact on the labor market in Bulgaria include the still manifesting impact of major structural changes in regional economies, related to the closure of a number of productive regions, with the inconsistency of the education system, in particular the vocational education, by labor demand characteristics, the highly uneven distribution of economic activity, even within the same field, ilnost, unfair practices so employers and others. The higher education system is highly fragmented and polarized throughout the country. This process of stimulating the labor market requires the implementation of an active policy involving the development and implementation of a number of training and education programs, especially of those at risk, in order to get out of the long-term unemployed category and to be given the opportunity implementation, thereby contributing to the achievement of sustainable regional development based on knowledge and innovation [4].

The total number of universities and colleges is 54, of which 24 are concentrated in the capital. This number far exceeds the average of a number of EU and European countries 
comparable in population and territory. At the same time, the number of students studying at universities and colleges is decreasing due to demographic factors, the declining quality of university education and the preference of young people to study abroad. In the academic 2018/2019 year, the total number of students in Bulgaria enrolled in the four educational-qualification degrees ('Professional bachelor', 'Bachelor', 'Master' and 'Doctor') was 229.5 thousand, which is with 6.9 thousand, or $2.9 \%$ less compared to the previous academic year. During the 2018/2019 academic year, for acquiring of the degree 'Professional bachelor', in colleges were enrolled 8181 students. In comparison with the previous year their number decreased with 346 or with $4.1 \%$. Out of the total number of students in colleges, 1501 or $18.3 \%$ were enrolled in private colleges [1].

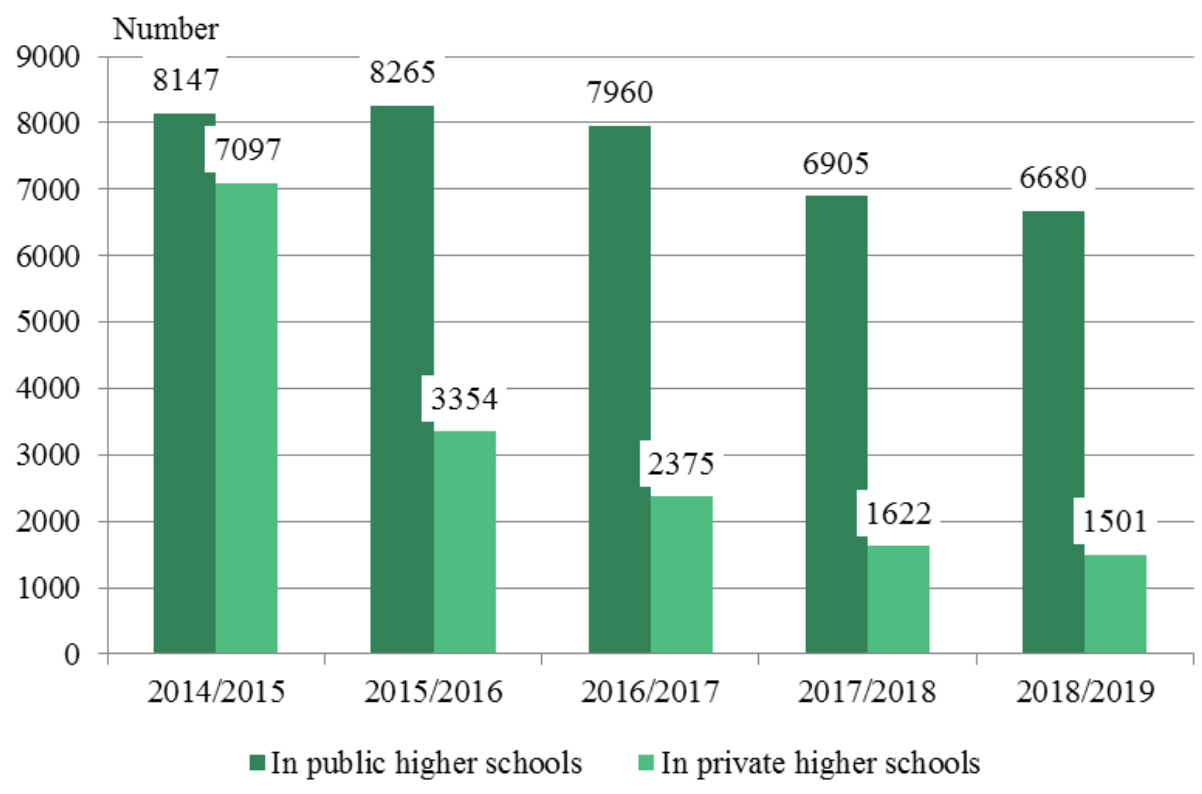

Figure 1. Students in colleges

Source: NSI, Bulgaria

The wide range of specialties in higher education is not always in line with the needs of the real national economy and the needs of the labor market in our country. At the same time, many university graduates go to work in EU countries, further reducing the effectiveness of investment in the system. In 2018/2019 academic year, for acquiring educational-qualification degrees 'Bachelor' and 'Master' in universities and specialized higher schools were enrolled 214.8 thousand students and in comparison with the previous year their number decreased by 6.4 thousand or by $2.9 \%$. In private educational institutions were enrolled 26.8 thousand students or $12.5 \%$ of the total number enrolled in educationalqualification degrees 'Bachelor' and 'Master' [1].

Therefore, higher education funding for higher education aims to improve its quality and applicability in the labor market. Nearly half of the companies that hired employees immediately after completing their secondary or tertiary education found gaps in their preparation. Persons who have started work after graduation have a serious deficit in their professional knowledge and skills, in their ability to work independently and to make decisions (about $70 \%$ of companies indicate a lack of the first type of skills, and about $60 \%$ - the second type). About $40 \%$ of companies believe that young people cannot work as a team. At the same time, only $17 \%$ of public sector employers found gaps in the preparation of young people with secondary education, while this finding was made by 
over $80 \%$ of private enterprises. About $15 \%$ of public-sector enterprises believe that high school graduates cannot work independently and make decisions, while over $70 \%$ of those in the private sector are of the same opinion [2].

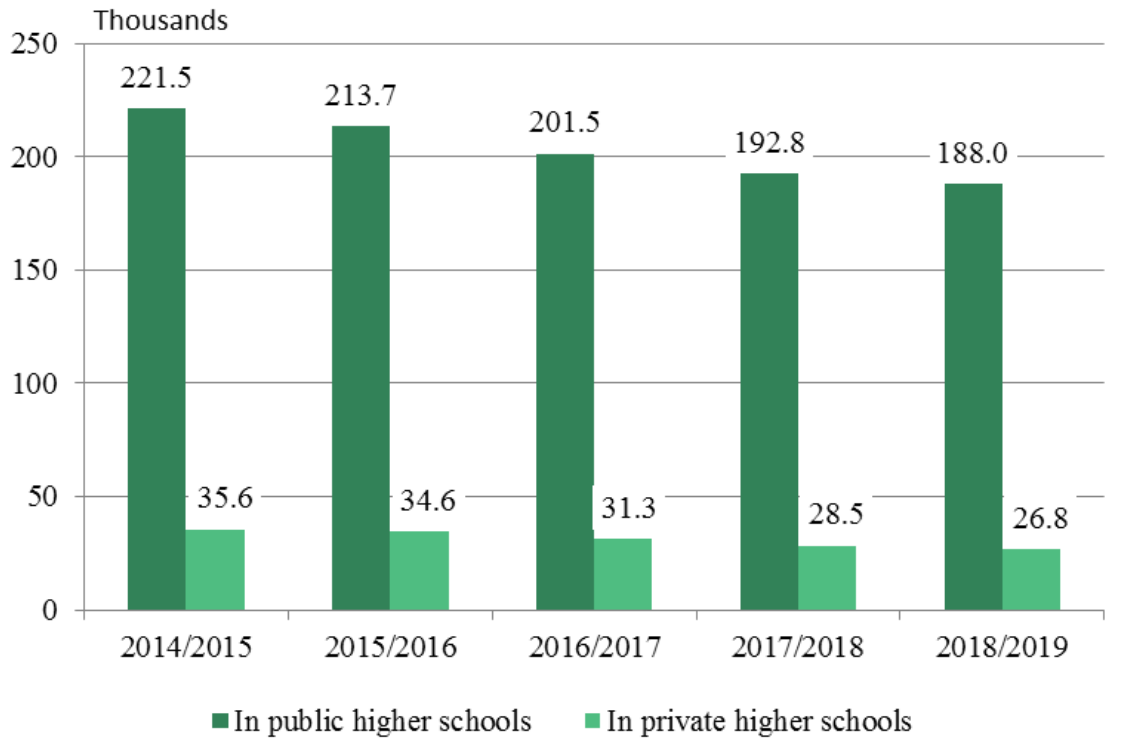

Figure 2. Students in universities and specialized higher schools Source: NSI, Bulgaria

Another important element of social regional development is health. The life expectancy in the country, although growing steadily, remains the lowest in the EU. Compared to 2010 , it increased by one year and reached 74.8 years in 2017 , while maintaining the differences between the Northwest region of 73.5 years and the Southwest region - 75.7 years. Serious risk factors (smoking, alcohol use, obesity, unbalanced diet and low motor activity) continue to affect a large part of the population and endanger its health. Raising the health culture, especially among minority groups characterized by limited access to health services, is a major challenge [2].

In 2017, the highest infant mortality in the EU was registered in Malta and Romania (6.7 per 1000 births), followed by Bulgaria with 6.4 deaths per 1000 births. A positive trend is the continuing decline in the infant mortality rate in the country. The infant mortality rate of 6.4 reached in 2017 is the lowest in the history of Bulgaria's demographic statistics. This indicator shows a long-term downward trend - in 2000 the infant mortality rate was $13.3 \%$, in 2010 - $9.4 \%$, and in 2015 - $6.6 \%$, but still higher than in the EU ( $3.6 \%$ ). Despite the decreasing trend in mortality in other age groups, Bulgaria still has a higher mortality rate $(15.5 \%$ ) compared to EU countries $(10.2 \%$ ). In the statistical regions with the lowest infant mortality, the Southwestern region stands out with a coefficient of 3.4 $\%$, and with the worst values is the Southeast region with $8.8 \%$. In regional terms, in 2017 in a total of eleven districts the infant mortality rate was lower than the national average, with the lowest being in the districts of Vidin - $1.7 \%$ and Sofia (capital) - 2.6 $\%$. The highest values of the infant mortality rate were registered in the districts of Dobrich (12.9\%o), Sliven (12.6,), Pazardzhik (11.1), where infant mortality is a serious problem, which speaks of a low health culture among the population, most -more among ethnic minorities. Healthcare facilities and hospital beds are unevenly distributed throughout the country, which does not provide equal access to health services for people, 
including those living in small, remote cities and towns [5]. This finding calls for a welltargeted policy on health infrastructure and a clearer outline of settlements where access to general and specialized health care is difficult. The reduction and aging of the population and the depopulation and impoverishment of the national periphery, in addition to poorly maintained transport infrastructure to remote settlements, are serious challenges for both health and regional policy. 27 regional emergency centers are located in the regional centers. Together with 173 branches, they cover the entire national territory and are a guarantee of quick response and a 30-minute isochron is provided within the territory served. According to the Emergency Card, some of the branches serve the territory of several municipalities, but there are cases in which several branches serve the population of one municipality, depending on the size of the municipalities. By the end of 2016, Bulgaria was meeting 11 of the indicators related to health enhancing physical activity and was working to reach the values of another 10 indicators, ahead of Cyprus and Portugal. Similarly to most EU Member States, the low efficiency of the implemented measures for increasing the physical activity of the population from different age groups is taken into account. The training of children with sports talents takes place in 24 sports schools, 6 of which are state and 18 municipal, 19 of which are located in the district centers. The state sports schools were set up in the big cities - Sofia, Ruse, Varna, Burgas, Plovdiv and Pleven. Three of the municipal sports schools are located in smaller cities, but with sports traditions - Chepelare, Samokov and Sandanski, in Sofia the sports schools are 4. In the 2019/2020 school year, a total of 1758 students will be trained in 65 classes, while in the 2016/2017 school year, a total of 1741 students will be trained in 64 classes. The territorial distribution of this infrastructure follows the trends in population distribution and is concentrated in the Southwestern region, where 53 sites are located. There are at least six gyms and gyms in the Northwestern region. The territorial disproportions are more pronounced at the district level, and in 4 districts from Northern Bulgaria there are no registered gyms and complexes (Vratsa, Razgrad, Silistra and Targovishte). Most sites are registered in the districts of Sofia (capital), Plovdiv and Varna [2].

Changes in the social and economic situation in Bulgaria after 1989 led to changes in the reproductive behaviour of the population. The transition from centrally planned economy to market economy and the accompanying economic crisis created conditions for a rapid drop in the economic activity of the population and an increase in unemployment [7].

The year 2017 ended with good results for the Bulgarian labor market, similar to the previous two - unemployment reached a new record low, employment is going up, and labor income is also continuing to grow steadily. In 2017, over half of the country's workforce was located in the Southwest and South Central regions, and the smallest in the Northwest region (9.3\%). The economic activity rate for the period 2014-2017 increased by 2.3 percentage points from $69.0 \%$ to $71.3 \%$. It is the highest in the Southwestern region (74.6\%) and lowest in the Northwestern $(65.4 \%)$ and in the North Central Region (68.5\%). The employment rate is a leading indicator of the labor market, showing the share of the working population. Most of the labor market challenges of the previous year continued this year. The lack of quality workforce and the growing mismatch in the skills sought and offered continued to weigh on the Bulgarian labor market. The employment of the most active population (15-64 years) marked a significant growth at the national level in 2014-2017, reaching 66.9\% in 2017 and almost catching up with the average employment rates for the EU - 67.6\%. Currently, employment has surpassed pre-crisis levels since 2008, when the employment rate was 64\%. The 
employment rate in cities (70.0\%) is higher than in rural areas $(57.4 \%)$, as well as in men (70.1\%), compared to women $(63.1 \%)$. In 2017, the Southwestern region has the highest employment rate $(72.1 \%)$. In practice, none of the old challenges in the labor market have been resolved this year, and they are expected to be carried forward to next year. In addition, the qualifications and skills of the workforce have not yet been adequately addressed and inevitably the pressure on the labor market will continue. The lowest employment rate is in the Northwestern region (57.9\%) and in the North Central region (63.7\%). At the regional level, employment in the strongest economic centers - the districts of Sofia (capital), Plovdiv and Stara Zagora already exceeds 70\%. There are serious lags in the districts of Vidin, Vratsa, Lovech, Montana, Razgrad, Silistra and Sliven. The difference between employment rates in the best-represented district of Sofia (capital) and the least-favored district of Vratsa is 19.3 percentage points. The growing mismatch between labor demand and available resources is driving businesses to "import" labor, with some companies even considering expansion abroad because of the inability to find the right specialists. The unemployment rate of the population aged 15-64 in the country in 2017 was $6.2 \%$, while in 2014 it was 5.3 percentage points higher. The levels of unemployment achieved by region show an increase in regional disparities. In the Northwestern region the values of the indicator are $11.4 \%$, in the Northeast $-9.4 \%$, while in the Southwestern region it is only 3.3\%. Youth unemployment (15-24 years) is improving, reaching $12.9 \%$ in 2017 and lower than the EU average (16.8\%). However, the positive dynamics can only partly be explained by the increase in employment among young people over the period considered, while the declining addition of economically active persons in this age group is crucial [2]. The aging population in Bulgaria poses many challenges for policy makers in terms of employment, working conditions, standard of living and well-being. This has led to concerns about the sustainability of pension systems and labor supply. Promoting job opportunities for an aging workforce requires new thinking at business, national and European level. According to the socio-economic characteristics of the unemployed, age and education are again the social prerequisites for getting out of unemployment, leaving the problem of long-term unemployed who find it difficult to make a transition to employment. Their relative share of the total unemployed remained at around [3].

\section{CONCLUSION}

In conclusion higher education is strongly influenced by demographic factors. The decreasing number of students enrolled in the system of pre-school and school education, the changing age structure of the population necessitates a rethinking of the model for the development of the educational system in all its forms and territorial dimensions. It must ensure equal access for all ages and social groups to various forms of education and training in the outermost regions of the country. Changes in education policy will be directed towards introducing more innovative teaching methods, linking curricula and curricula with current topics and the needs of the local economy, integrating Roma and students with special needs in the education system. Higher education needs to improve links with research and innovation centers, including abroad, with businesses and with the needs of the labor market. It is envisaged that priority will be given to funding for fields of science, technology, engineering and mathematics (STEM), especially ICTs and mathematics, at public universities. A serious problem in health care is the remoteness of some small settlements from the place of medical assistance (primary, specialized, hospital, emergency and emergency). A significant potential for improving the efficiency 
of the system is the entry of e-governance into the healthcare system. Bulgaria lags behind this indicator and is ranked 23rd in the EU. The downward trend in the incoming staff in the healthcare system is worrying due to the reduced number of students and the emigration of qualified medical specialists (doctors and nurses). In the medium to long term, the combination of these processes threatens to compromise the ability of the system to provide quality and timely health services to the population. Important healthcare tasks will be linked to the demographic projections and changing needs of the population of all age groups, who need to be prepared for longer life expectancy through appropriate preventive and educational care. There are serious problems in the management, efficient use and maintenance of the built base, in the overall renovation of the equipment, in the provision of well-trained staff and high quality of services. Equal access to sports and sports facilities and facilities for vulnerable groups, people with disabilities and people with disabilities has not yet been ensured. There are settlements where sports facilities are lacking, the pedestrian and bicycle traffic network is not developed. According to Eurostat population estimates in EU Member States, the number of people of working age in Bulgaria will continue to decline in the future, and this is the main factor that will influence the reduction of the workforce in the country. According to the forecasts for the demand and supply of labor at the European Center for the Development of Vocational Education by 2025, both the workforce in Bulgaria and employment are projected to decline. The decrease will continue until 2050. The negative employment trend will be mainly due to the decrease in the number of employed persons with primary and lower education. There will be a decrease in the number of employees with secondary education. For the period up to 2025, employment will only increase in higher education. These trends will lead to a change in the structure of employment, increasing the relative share of the workforce with tertiary education and reducing the share of people with lower educational attainment. This trend will continue until 2050. The labor market in our country is catching up due to the growing mismatch in the skills and capabilities of the workforce and the needs of the economy. And while there are policies that partly attempt to change, the problem of quality of workforce is escalating but not adequately addressed, economists and financiers say.

\section{REFERENCES}

[1] Education in the republic of Bulgaria for 2018/2019 school year. NSI

[2] Socio-economic analysis of the regions in the republic of Bulgaria, 2019

[3] Ivanov M. Peculiarities in the socio-economic development of the regions in Bulgaria, Journal of mining and geological sciences, Vol. 60, Part IV, Humanitarian sciences and Economics, 2017

[4] Ivanov M. Problems in the labor market in Bulgaria, Journal: Contemporary Management Practices X, 2019

[5] Atanasova A., Naydenov K. (2020) The Innovative Approaches for the Development of Smart Cities. In: Nedkov S. et al. (eds) Smart Geography. Key Challenges in Geography (EUROGEO Book Series). Springer, Cham

[6] Traykov T. Kl. Naydenov Demographic situation in rural areas of republic of Bulgaria in 21 century, International Scientific Conference GEOBALCANICA 2015, DOI: http://dx.doi.org/10.18509/GBP.2015.26

[7] Traykov T. K. Tsvetkov Trends and specifics in the reproductive behaviour of the bulgarian population at the beginning of the 21-st century, International Conference "Smart Geography", Abstracts p. 277-288, At: Sofia, Bulgaria, 2018 